\title{
Meta-Analysis of Randomized Controlled Trials on the Efficacy of Di’ao Xinxuekang Capsule and Isosorbide Dinitrate in Treating Angina Pectoris
}

\author{
Yongliang Jia,, ${ }^{1,2}$ Cong Chen, ${ }^{1,2}$ Choi-San Ng, ${ }^{1,2}$ and Siu-Wai Leung, 2,3 \\ ${ }^{1}$ State Key Laboratory of Quality Research in Chinese Medicine, University of Macau, Taipa 999078, Macau \\ ${ }^{2}$ Institute of Chinese Medical Sciences, University of Macau, Taipa 999078, Macau \\ ${ }^{3}$ BIGHT Laboratory, School of Informatics, University of Edinburgh, Edinburgh EH8 9AB, UK
}

Correspondence should be addressed to Siu-Wai Leung, siu@inf.ed.ac.uk

Received 1 November 2011; Revised 20 December 2011; Accepted 20 December 2011

Academic Editor: Gerhard Litscher

Copyright ( $) 2012$ Yongliang Jia et al. This is an open access article distributed under the Creative Commons Attribution License, which permits unrestricted use, distribution, and reproduction in any medium, provided the original work is properly cited.

\begin{abstract}
Objective. Randomized controlled trials (RCTs) on di'ao xinxuekang capsule (XXK) in treating angina pectoris were published only in Chinese and have not been systematically reviewed particularly for comparing XXK with isosorbide dinitrate (ISDN). This study aims to provide a comprehensive PRISMA compliant and internationally accessible systematic review and meta-analysis to evaluate the efficacies of XXK and ISDN in treating angina pectoris. Methods. The RCTs published between 1989 and 2011 on XXK and ISDN in treating angina pectoris were selected according to specific criteria. Meta-analysis was performed to evaluate the symptomatic (SYMPTOMS) and electrocardiographic (ECG) improvements after treatment. Odds ratios (OR) were used to measure effect sizes. Subgroup analysis, sensitivity analysis, and metaregression were conducted to evaluate the robustness of the results. Results. Seven RCTs with 550 participants were eligible. Overall ORs for comparing XXK with ISDN were 4.11 (95\% CI: $2.57,6.55)$ in SYMPTOMS and 2.37 (95\% CI : 1.46, 3.84) in ECG. Subgroup analysis, sensitivity analysis, and metaregression found no significant dependence of overall ORs upon specific study characteristics. Conclusion. The meta-analysis of eligible but limited RCTs demonstrates that XXK seems to be more effective than ISDN in treating angina pectoris. Further RCTs of high quality are warranted to be conducted for update of the results of this meta-analysis.
\end{abstract}

\section{Introduction}

Ischemic heart disease, an imbalance coronary blood flow and myocardial requirement [1], usually has symptomatic discomfort in the chest, that is, angina pectoris [2], due to coronary insufficiency and myocardium ischemia and hypoxia [3]. Nitrates, calcium channel blockers, beta-receptor blockers are common drugs for treating angina pectoris [4]. Isosorbide dinitrate (ISDN) is one of the most effective and frequently used nitrates for treating angina pectoris $[3,5]$. Di'ao xinxuekang (XXK) capsule is a second-class new drug of China for treating angina pectoris [6]. The main components of XXK are extracted from the rhizomes of Dioscorea panthaica Prain et Burkill and Dioscorea nipponica Makino [7].

Randomized controlled trials (RCTs) on treatments of angina pectoris found that XXK was more effective than aspirin [8] and more effective than some Chinese medicinal formulations including compound danshen, guanxin suquan pills, and danshen dripping pills $[9,10]$. A previous systematic review published in 2010 [11] suggested that XXK were more effective than danshen tablet but did not cite any eligible RCT and did not report any subgroup or sensitivity analysis. XXK and danshen dripping pills are two popular Chinese medicinal products often compared with ISDN in treating angina pectoris [12-15]. A recent systematic review published in 2011 [16] indicated that danshen dripping pills was more effective than ISDN. Furthermore, there is no systematic review or meta-analysis of RCTs comparing XXK with conventional nitrates such as ISDN. As such, it is not clear about whether XXK is more effective than ISDN in treating angina pectoris. This study aims to conduct a comprehensive, PRISMA-compliant [17], internationally 
accessible, and timely meta-analysis, with subgroup and sensitivity analyses of RCTs to compare the efficacies of XXK and ISDN in treating angina pectoris.

\section{Methods}

2.1. Study Selection Criteria. RCTs published in Chinese or English between 1989 (i.e., the year of XXK launch) and 2011 on comparing XXK with ISDN in treating angina pectoris were screened. Inclusion criteria were as follow: (a) participants were suffering from and being treated for angina pectoris; (b) the study was claimed to be an RCT; (c) the study compared XXK with ISDN in efficacy; (d) duration of treatment (follow-up period) was at least four weeks.

Exclusion criteria were as follow: (a) duplicated or redundant study; (b) XXK was used in combination with other drugs; (c) ISDN was used in combination with other drugs; (d) the study did not include symptomatic (SYMPTOMS) improvement as the major outcome.

2.2. Search Strategies. RCT reports published between the 1989 and 2011 were searched from databases including China National Knowledge Infrastructure (CNKI), WanFang Data, China Master Theses Full-text Database (CMTD), China Doctor Dissertations Full-text Database (CDMD), Medline, PubMed, Cochrane Library, ScienceDirect and EMbase. The last search was performed on 22 August 2011.

The search strategy was formulated mainly for searching xinxuekang because of few publications on the drug. For instance, WanFang Data was searched with such a simple query as: ("guanxinbing (heart disease)" in title or "xinjiaotong (angina pectoris)" in title) and "xinxuekang" in abstract). The exact search strategies and query syntax were slightly adjusted according to the users' instructions from different databases.

2.3. Study Selection. Two reviewers (Y. Jia and C. Chen) independently screened all retrieved studies according to the eligibility criteria. Disagreement between reviewers was resolved by consensus. A flow diagram of study selection was generated according to the PRISMA requirements [17].

2.4. Data Extraction. Two reviewers (Y. Jia and C. Ng) independently extracted data items comprised (a) publication year; (b) number of authors; (c) trial dates; (d) baseline comparizson of participants between groups; (e) sample size; (f) outcome measures; (g) dosage and treatment duration; (h) incidence of adverse events (AE).

2.5. Quality Assessment of Included Studies. Two reviewers (Y. Jia and C. Chen) independently assessed the quality of the included studies according to the Jadad scale [18], the M scale [16] and the Cochrane risk of bias tool [19]. The Jadad scale focused on three criteria including "randomization," "blinding," and "dropouts" for assessing the quality of RCT. The M scale was modified from the Jadad scale with two additional criteria "baseline comparison of participants" and "adverse event report." The Cochrane risk of bias tool was designed for more detailed assessment including criteria for (a) allocation concealment, (b) blinding of participants, (c) personnel and outcome assessment, (d) incomplete outcome data, (e) selective reporting, and (f) other biases [19].

2.6. Criteria for Symptomatic and ECG Improvements. Effective symptomatic improvements should achieve at least 50\% (basic) or $80 \%$ (significant) reduction in frequency of feeling angina chest pain. Effective ECG improvements should achieve at least $0.05 \mathrm{mV}$ lowering at ST segment in ECG (basic) or nearly normal (significant) ECG during an exercise test as suggested in the ACC/AHA guideline [3].

2.7. Meta-Analysis. Effect sizes were presented by odds ratios (OR) [20] and 95\% confidence intervals (CI) [21]. Overall meta-analysis and subgroup analysis employed the randomeffects model because the homogeneity of the studies could not be guaranteed. Heterogeneity among studies was assessed by chi-squared $\left(\chi^{2}\right)$ and $I$-squared $\left(I^{2}\right)[22]$.

2.8. Subgroup and Sensitivity Analysis. Subgroup analysis was conducted to evaluate the overall effects in possible subgroups which were grouped according to the characteristics of the studies, including year of publication, number of authors, follow-up period, sample sizes, study periods, quality of the studies (M score), and improvements in SYMPTOMS and ECG. Mann-Whitney-Wilcoxon test was used to analyze the difference between subgroups. Sensitivity analyses were performed after excluding some studies to assess the influence of such studies on the overall result of this metaanalysis.

2.9. Metaregression and Risk of Bias across Studies. Funnel plots [23], Begg's rank correlation test [24], and Egger's linear regression test [25] were employed to assess the publication bias. Metaregression [26] was conducted to find the possible relationship between the efficacy and the factors such as sample sizes, M score, years of publication, and quality of RCTs.

2.10. Adverse Events. AEs of RCTs including nonreported AEs, types and frequency of AEs reported were surveyed for incidence of AEs during XXK and ISDN treatments.

2.11. Statistical Analysis. Meta-analysis and forest plot generation were performed with Review Manager 5 [27]. Metaregression and funnel plot generation was conducted with Stata software version 11 (StataCorp LP, USA). Statistical software R [28] was used to perform conventional statistical analysis, including Spearman correlation and MannWhitney-Wilcoxon unpaired two-sample test. $P$ values lower than 0.05 were considered statistically significant.

\section{Results}

3.1. Study Selection. The process of study selection was depicted in Figure 1. The database search found 435 records, 


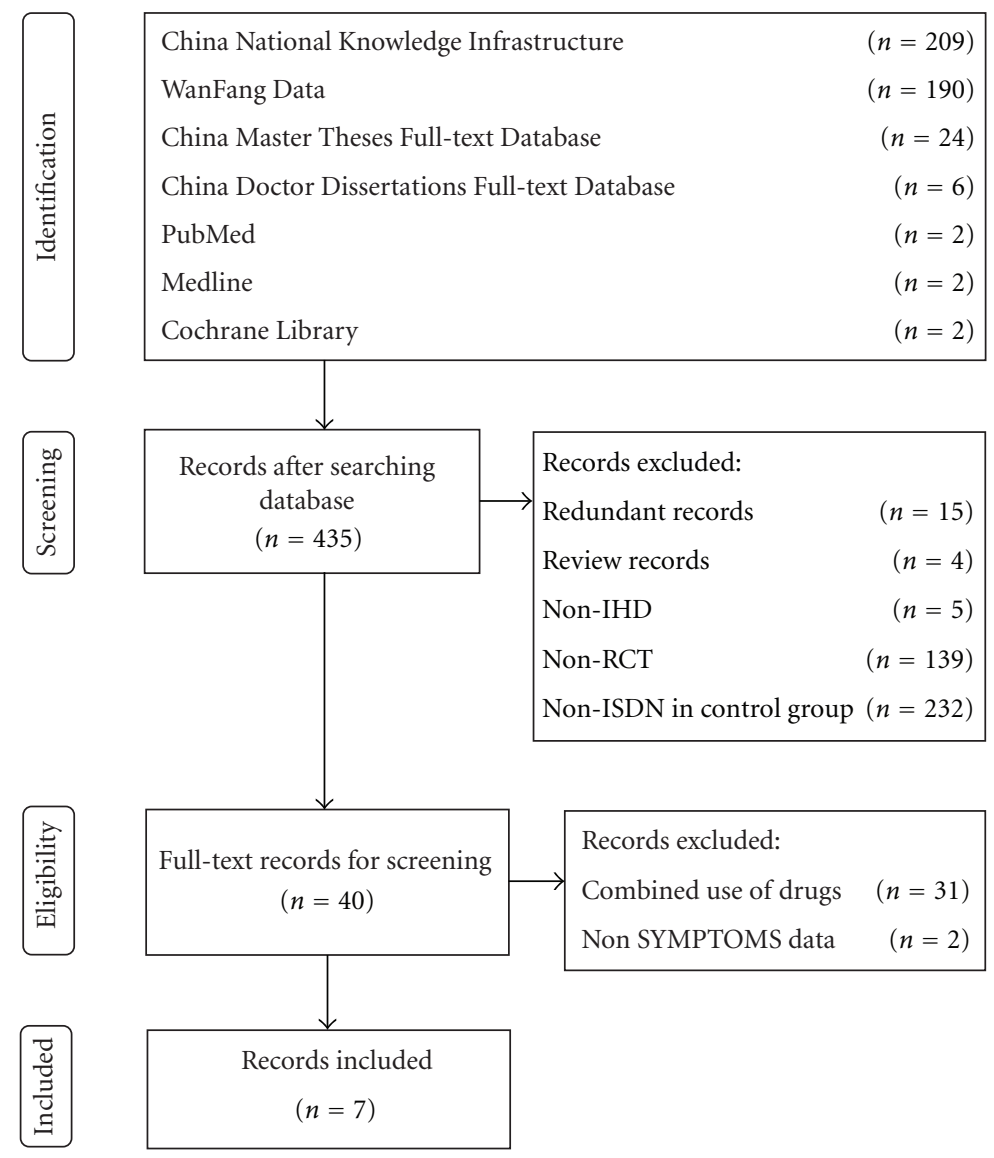

FIGURE 1: Process of searching and screening studies.

TABLE 1: Summary of the included studies evaluating the efficacy of XXK in treating angina pectoris.

\begin{tabular}{|c|c|c|c|c|c|c|c|c|c|c|c|}
\hline Study & $\begin{array}{c}\text { No. of } \\
\text { authors }\end{array}$ & $\begin{array}{l}\text { Trail } \\
\text { date }\end{array}$ & $\begin{array}{c}\text { Sample } \\
\text { size }\end{array}$ & $\begin{array}{c}\text { Follow-up } \\
\text { period }\end{array}$ & Comparable & AEs & $\begin{array}{l}\text { Outcome } \\
\text { measures }\end{array}$ & $\begin{array}{c}\text { XXK daily } \\
\text { dosage }\end{array}$ & $\begin{array}{c}\text { ISDN daily } \\
\text { dosage }\end{array}$ & $\begin{array}{l}\text { Jadad } \\
\text { score }\end{array}$ & M score \\
\hline Zeng et al. [29] & 6 & 0 & 34 & 28 days & 1 & 1 & SYM, ECG & 6 pills & $40 \mathrm{mg}$ & 2 & 4 \\
\hline Dai [30] & 1 & 1 & 120 & 28 days & 0 & 0 & SYM & 6 pills & $30 \mathrm{mg}$ & 2 & 2 \\
\hline $\mathrm{Hu}[31]$ & 1 & 0 & 84 & 28 days & 1 & 1 & SYM, ECG & 6 pills & $30 \mathrm{mg}$ & 2 & 4 \\
\hline Li and Zhang [34] & 2 & 1 & 60 & 28 days & 0 & 1 & SYM & 6 pills & $60 \mathrm{mg}$ & 2 & 3 \\
\hline $\mathrm{Qu}[32]$ & 1 & 1 & 60 & 28 days & 1 & 0 & SYM, ECG & 6 pills & $30 \mathrm{mg}$ & 2 & 3 \\
\hline Shen [33] & 1 & 1 & 132 & 28 days & 0 & 1 & SYM, ECG & 6 pills & $30 \mathrm{mg}$ & 2 & 3 \\
\hline Zhu et al. [35] & 5 & 1 & 60 & 28 days & 1 & 0 & SYM, ECG & 6 pills & $30 \mathrm{mg}$ & 2 & 3 \\
\hline
\end{tabular}

XXK is di'ao xinxuekang capsule; ISDN is isosorbide dinitrate; SYM is SYMPTOMS; ECG is electrocardiogram; AEs is adverse events; Numbers 1 and 0 in "Trial date" mean that study reported or did not report the trial date, respectively; Numbers 1 and 0 in "Comparable" mean that the study reported the comparability between the experiment and control groups (1) or the study did not report that (0).

including 209 records from CNKI, 190 records from WanFang Data, 24 records from CMTD, 6 records from CDMD, 2 records from PubMed, 2 records from Medline, and 2 records from the Cochrane Library. According to the prespecified selection criteria described in the section Methods, only seven studies [29-35] were found eligible for further quality assessment and meta-analysis.

3.2. Study Characteristics. Table 1 lists the main characteristics of the included studies. All of the studies were published in Chinese language between 1993 and 2007 with a total of
550 participants. The mean sample size was 78.6 (median $=$ 60.0; 95\% CI: 52.2, 105.0). The follow-up periods were all 28 days. The daily dosage of XXK was 6 pills in all studies. The daily dosages of ISDN were $30 \mathrm{mg}$ in five studies, $40 \mathrm{mg}$ and $60 \mathrm{mg}$ in the other two studies. All seven included studies reported SYMPTOMS changes as outcome measures while five studies [29, 31-33,35] also reported ECG changes.

3.3. Quality Assessment of Included Studies. Table 1 lists the results of quality assessment according to the Jadad and $\mathrm{M}$ scale. All included studies were scored 2 according to 


\begin{tabular}{|c|c|c|c|c|c|c|c|c|c|c|}
\hline \multirow{2}{*}{$\begin{array}{l}\text { Study or subgroup } \\
\text { Dai, } 2000\end{array}$} & \multicolumn{2}{|c|}{$\begin{array}{c}\text { XXK } \\
\text { Events Total }\end{array}$} & \multicolumn{2}{|c|}{$\begin{array}{r}\text { ISDN } \\
\text { Events Total }\end{array}$} & \multirow{2}{*}{$\frac{\text { Weight }}{29.6 \%}$} & \multirow{2}{*}{$\begin{array}{c}\text { Odds ratio } \\
\text { M-H, random, 95\% CI }\end{array}$} & \multicolumn{4}{|c|}{$\begin{array}{c}\text { Odds ratio } \\
\mathrm{M}-\mathrm{H} \text {, random, } 95 \% \mathrm{CI}\end{array}$} \\
\hline & 45 & 60 & 18 & 60 & & & & & $=$ & \\
\hline $\mathrm{Hu}, 2009$ & 38 & 43 & 25 & 41 & $16.1 \%$ & $4.86[1.58,14.97]$ & & & & \\
\hline $\mathrm{Li}, 2000$ & 29 & 30 & 24 & 30 & $4.5 \%$ & $7.25[0.82,64.46]$ & & & & \\
\hline Qu, 2006 & 28 & 30 & 26 & 30 & $6.7 \%$ & $2.15[0.36,12.76]$ & & & & \\
\hline Shen, 1993 & 59 & 66 & 46 & 66 & $22.3 \%$ & $3.66[1.43,9.41]$ & & & & \\
\hline Zeng, 1996 & 12 & 16 & 14 & 18 & $8.4 \%$ & $0.86[0.18,4.19]$ & & & & \\
\hline Zhu, 2007 & 26 & 30 & 19 & 30 & $12.5 \%$ & $3.76[1.04,13.65]$ & & & & \\
\hline Total $(95 \%$ CI $)$ & & 275 & & 275 & $100 \%$ & $4.11[2.57,6.55]$ & & & & \\
\hline Total events & 237 & & 172 & & & & & & & \\
\hline \multicolumn{6}{|c|}{ Heterogeneity: $\tau^{2}=0.02 ; \chi^{2}=6.36, d f=6(P=0.38) ; I^{2}=6 \%$} & & 0.02 & 0.1 & 10 & 50 \\
\hline \multicolumn{6}{|c|}{ Test for overall effect: $Z=5.93(P<0.00001)$} & & \multicolumn{4}{|c|}{ ISDN XXK } \\
\hline
\end{tabular}

FIGURE 2: Forest plot of outcome measure SYMPTOMS.

\begin{tabular}{|c|c|c|c|c|c|c|c|c|}
\hline \multirow{2}{*}{$\begin{array}{l}\text { Study or subgroup } \\
\mathrm{Hu}, 2009\end{array}$} & \multicolumn{2}{|c|}{$\begin{array}{c}\text { XXK } \\
\text { Events Total }\end{array}$} & \multicolumn{3}{|c|}{ ISDN } & $\begin{array}{c}\text { Odds ratio } \\
\mathrm{M}-\mathrm{H} \text {, random, } 95 \% \mathrm{CI}\end{array}$ & \multicolumn{2}{|c|}{$\begin{array}{c}\text { Odds ratio } \\
\mathrm{M}-\mathrm{H} \text {, random, } 95 \% \mathrm{CI}\end{array}$} \\
\hline & 37 & 43 & 28 & 41 & $19.8 \%$ & $2.86[0.97,8.47]$ & & $5-1$ \\
\hline Qu, 2006 & 28 & 30 & 24 & 30 & $8.2 \%$ & $3.5[0.65,18.98]$ & & \\
\hline Shen, 1993 & 34 & 66 & 18 & 66 & $44.3 \%$ & $2.83[1.37,5.85]$ & & 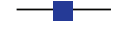 \\
\hline Zeng, 1996 & 9 & 16 & 11 & 18 & $12.5 \%$ & $0.82[0.21,3.22]$ & & \\
\hline Zhu, 2007 & 25 & 30 & 21 & 30 & $15.2 \%$ & $2.14[0.62,7.39]$ & & \\
\hline \multicolumn{2}{|l|}{ Total $(95 \% \mathrm{CI})$} & 185 & & 185 & $100 \%$ & $2.37[1.46,3.84]$ & & \\
\hline Total events & \multicolumn{2}{|l|}{133} & 102 & & & \multirow{2}{*}{$\stackrel{\longmapsto}{0.05}$} & & \\
\hline \multicolumn{6}{|c|}{ Heterogeneity: $\tau^{2}=0 ; \chi^{2}=2.9, d f=4(P=0.57) ; I^{2}=0 \%$} & & 0.2 & 5 \\
\hline \multicolumn{6}{|c|}{ Test for overall effect: $Z=3.5(P<0.0005)$} & \multicolumn{3}{|c|}{ ISDN XXK } \\
\hline
\end{tabular}

Figure 3: Forest plot of outcome measure ECG.

the Jadad scale (between 0 and 5 points), in consistency with the average quality of Chinese RCTs [36].

According to M scale, four RCTs scored three, two RCTs scored two, and one RCT scored one. Four included studies $[29,31,32,35]$ reported baseline comparison of participants in experiment and control group. Three included studies [30, $32,35]$ did not report AEs. One included study [29] reported types of AEs. Three included studies [31, 34, 35] reported types and number of AEs.

Cochrane risk of bias tool also gave similar ratings, that is, (a) unclear bias in random sequence generation; (b) high bias in allocation concealment; (c) high bias in blinding of participants; (d) personnel and outcome assessment; (e) low bias in incomplete outcome data; (f) low bias in selective reporting; (g) unclear bias in other bias.

3.4. Individual Studies and Their Synthesis. As shown in Figure 2, the overall OR of SYMPTOMS was $4.11(95 \% \mathrm{CI}=$ 2.57-6.55, $Z=5.93, P<0.00001)$ with a heterogeneity $\left(\tau=0.02, I^{2}=6 \%, P=0.38\right)$ among the seven studies with SYMPTOMS outcome. Figure 3 shows that the overall OR of ECG was 2.37 (95\% CI $=1.46-3.84, Z=3.50$, $P=0.0005)$ with a heterogeneity $\left(\tau=0, I^{2}=0 \%, P=\right.$ 0.57 ) among the five studies with ECG outcome. Both the ORs of SYMPTOMS and ECG indicated that XXK is more effective than ISDN in treating angina pectoris. The overall
OR of SYMPTOMS was bigger than that of ECG although the correlation in ORs between SYMPTOMS and ECG was not statistically significant $(P=0.6833)$.

3.5. Subgroup and Sensitivity Analysis. Based on study characteristics including $\mathrm{M}$ score $(\leq 3$ or $>3$ ), years of publication (before or after 1 January 2005), number of authors (1 or $>1$ ), dates of trial (reported or non-reported), baseline comparison of participants (yes or no), sample sizes $(<78$ or $\geq 78$ ), and reports of AEs (yes or no), subgroup analysis or sensitivity analysis were performed and their results are shown in Tables 2 and 3. Wilcoxon test for comparing the ORs between subgroups in terms of SYMPTOMS and ECG found no statistically significant differences.

When the improvements in SYMPTOMS were raised to a significant level from the basic level, the results remain effective and statistically significant $(\mathrm{OR}=1.83,95 \% \mathrm{CI}=$ $1.20-2.82, Z=2.77, P=0.006)$. The overall ECG OR decreased from 2.37 to 1.60 (95\% CI $=0.98-2.60, Z=1.88$, $P=0.06)$ with a heterogeneity $\left(\tau=2.38, I^{2}=0 \%, P=0.67\right)$. The results indicated there was not heterogeneity. Likewise, results of Spearman correlation analysis indicated there was not statistical significance $(P=0.95)$ between them.

3.6. Metaregression. Table 4 shows the results of metaregression between $\log \mathrm{OR}$ and study characteristics. As 
TABLE 2: Subgroups and sensitivity analysis based on ORs of SYMPTOMS outcomes.

\begin{tabular}{|c|c|c|c|c|c|c|c|c|c|c|c|}
\hline & Group & $\begin{array}{l}\text { No. of } \\
\text { studies }\end{array}$ & $\begin{array}{c}\text { No. of } \\
\text { participants }\end{array}$ & OR & Wilcoxon test & $95 \% \mathrm{CI}$ & $Z$ & $P($ effect $)$ & $I^{2}$ & $\chi^{2}$ & $P$ (het) \\
\hline \multirow[t]{2}{*}{ M score } & $\leq 3$ & 5 & 432 & 4.76 & $W=7$ & $2.85,7.95$ & 5.96 & $<0.00001$ & $0 \%$ & 2.21 & 0.70 \\
\hline & $>3$ & 2 & 118 & 2.24 & $P=0.4386$ & $0.41,12.17$ & 0.94 & 0.35 & $67 \%$ & 3.06 & 0.08 \\
\hline \multirow[t]{2}{*}{ Sample size } & $<78$ & 4 & 214 & 2.49 & $W=4$ & $1.09,5.68$ & 2.17 & 0.03 & $3 \%$ & 3.09 & 0.38 \\
\hline & $\geq 78$ & 3 & 336 & 5.22 & $P=0.4795$ & $3.05,8.93$ & 6.03 & $<0.00001$ & $0 \%$ & 1.07 & 0.59 \\
\hline \multirow[t]{2}{*}{ No. of authors } & 1 & 4 & 396 & 4.85 & $W=6$ & $2.90,8.11$ & 6.01 & $<0.00001$ & $0 \%$ & 1.94 & 0.58 \\
\hline & $>1$ & 3 & 154 & 2.61 & $P=1$ & $0.82,8.36$ & 1.62 & 0.11 & $35 \%$ & 3.06 & 0.22 \\
\hline \multirow[t]{2}{*}{ Publication year } & $<2005$ & 4 & 346 & 3.84 & $W=7$ & $1.65,8.93$ & 3.12 & 0.002 & $47 \%$ & 5.71 & 0.13 \\
\hline & $\geq 2005$ & 3 & 204 & 3.82 & $P=0.7237$ & $1.78,8.21$ & 3.44 & 0.0006 & $0 \%$ & 0.58 & 0.75 \\
\hline \multirow[t]{2}{*}{ Trial date } & Reported & 5 & 432 & 4.76 & $W=7$ & $2.85,7.95$ & 5.96 & $\begin{array}{c}<0.000 \\
01\end{array}$ & $0 \%$ & 2.21 & 0.70 \\
\hline & $\begin{array}{c}\text { Not } \\
\text { reported }\end{array}$ & 2 & 118 & 2.24 & $P=0.4386$ & $0.41,12.17$ & 0.94 & 0.35 & $67 \%$ & 3.06 & 0.08 \\
\hline \multirow[t]{2}{*}{ Comparable } & Reported & 4 & 238 & 2.83 & $W=2$ & $1.36,5.89$ & 2.78 & 0.005 & $10 \%$ & 3.35 & 0.34 \\
\hline & $\begin{array}{l}\text { Not } \\
\text { reported }\end{array}$ & 3 & 312 & 5.45 & $P=0.1573$ & $3.02,9.83$ & 5.64 & $<0.00001$ & $0 \%$ & 1.12 & 0.57 \\
\hline \multirow[t]{2}{*}{ AEs } & Reported & 4 & 310 & 3.30 & $W=6$ & $1.58,6.88$ & 3.18 & 0.0001 & $21 \%$ & 3.78 & 0.29 \\
\hline & $\begin{array}{c}\text { Not } \\
\text { reported }\end{array}$ & 3 & 240 & 5.17 & $P=1$ & $2.74,9.78$ & 5.06 & $<0.00001$ & $0 \%$ & 1.71 & 0.43 \\
\hline \multirow[t]{2}{*}{ Improvement } & $>50 \%$ & 7 & 550 & 4.11 & $W=7$ & $2.57,6.55$ & 5.93 & $<0.00001$ & $6 \%$ & 6.36 & 0.38 \\
\hline & $>80 \%$ & 5 & 370 & 1.83 & $P=0.2506$ & $1.20,2.82$ & 2.77 & 0.006 & $0 \%$ & 1.10 & 0.89 \\
\hline
\end{tabular}

CI is confidence interval. Mann-Whitney-Wilcoxon test was performed on ORs of SYMPTOMS. $Z$ and $P$ (effect) evaluated the statistics of overall effect; $I^{2}$, $\chi^{2}$, and $P$ (het) were computed to assess heterogeneity.

TABLE 3: Subgroups and sensitivity analysis based on ORs of ECG outcomes.

\begin{tabular}{|c|c|c|c|c|c|c|c|c|c|c|c|}
\hline & Group & $\begin{array}{l}\text { No. of } \\
\text { studies }\end{array}$ & $\begin{array}{c}\text { No. of } \\
\text { participants }\end{array}$ & OR & $\begin{array}{l}\text { Wilcoxon } \\
\text { test }\end{array}$ & $95 \%$ CI & $Z$ & $P($ effect $)$ & $I^{2}$ & $\chi^{2}$ & $P$ (het) \\
\hline \multirow[t]{2}{*}{ M score } & $\leq 3$ & 3 & 252 & 2.73 & $W=4$ & $1.52,4.91$ & 3.35 & 0.0008 & $0 \%$ & 0.24 & 0.89 \\
\hline & $>3$ & 2 & 118 & 1.65 & $P=0.5637$ & $0.49,5.57$ & 0.8 & 0.42 & $49 \%$ & 1.98 & 0.16 \\
\hline \multirow[t]{2}{*}{ Sample size } & $<78$ & 3 & 154 & 1.71 & $W=2$ & $0.77,3.84$ & 1.31 & 0.19 & $0 \%$ & 1.93 & 0.38 \\
\hline & $\geq 78$ & 2 & 216 & 2.84 & $P=0.5637$ & $1.56,5.20$ & 3.39 & 0.0007 & $0 \%$ & 0 & 0.99 \\
\hline \multirow[t]{2}{*}{ No. of authors } & 1 & 3 & 276 & 2.91 & $W=6$ & $1.65,5.14$ & 3.69 & 0.0002 & $0 \%$ & 0.05 & 0.97 \\
\hline & $>1$ & 2 & 94 & 1.39 & $P=0.0833$ & $0.54,3.55$ & 0.68 & 0.5 & $4 \%$ & 1.05 & 0.31 \\
\hline \multirow[t]{2}{*}{ Publication year } & $<2005$ & 2 & 166 & 1.75 & $W=1$ & $0.54,5.74$ & 0.93 & 0.35 & $60 \%$ & 2.47 & 0.12 \\
\hline & $\geq 2005$ & 3 & 204 & 2.69 & $P=0.2482$ & $1.29,5.60$ & 2.63 & 0.008 & $0 \%$ & 0.24 & 0.89 \\
\hline \multirow[t]{2}{*}{ Trial date } & Reported & 2 & 118 & 1.65 & $W=2$ & $0.49,5.57$ & 0.8 & 0.42 & $49 \%$ & 1.98 & 0.16 \\
\hline & Not reported & 3 & 252 & 2.73 & $P=0.5637$ & $1.52,4.91$ & 3.35 & 0.0008 & $0 \%$ & 0.24 & 0.89 \\
\hline \multirow[t]{2}{*}{ Comparable } & Reported & 4 & 238 & 2.06 & $W=2$ & $1.08,3.93$ & 2.19 & 0.03 & $0 \%$ & 2.48 & 0.48 \\
\hline & Not reported & 1 & 132 & 2.83 & $P=1$ & $1.37,5.85$ & 2.81 & 0.005 & NA & NA & NA \\
\hline \multirow[t]{2}{*}{ AEs } & Reported & 3 & 250 & 2.22 & $W=2$ & $1.14,4.32$ & 2.33 & 0.02 & $25 \%$ & 2.66 & 0.26 \\
\hline & Not reported & 2 & 120 & 2.54 & $P=0.5637$ & $0.94,6.90$ & 1.83 & 0.07 & $0 \%$ & 0.21 & 0.65 \\
\hline \multirow[t]{2}{*}{ Improvement } & $>50 \%$ & 5 & 370 & 2.37 & $W=14$ & $1.46,3.84$ & 3.5 & 0.0005 & $0 \%$ & 2.90 & 0.57 \\
\hline & $>80 \%$ & 5 & 370 & 1.60 & $P=0.7540$ & $0.98,2.60$ & 1.88 & 0.06 & $0 \%$ & 2.38 & 0.67 \\
\hline
\end{tabular}

CI is confidence interval. NA is not available; Mann-Whitney-Wilcoxon test was performed on ORs of ECG. $Z$ and $P$ (effect) evaluated the statistics of overall effect; $I^{2}, \chi^{2}$, and $P$ (het) were computed to assess heterogeneity.

the results of metaregression were not statistically significant, there seems to be no obvious correspondence of the efficacy to any study characteristic.

3.7. Risk of Bias across Studies. Publication biases were assessed by funnel plots (Figure 4), which found no obvious asymmetry for SYMPTOMS and ECG. The Begg's test (SYMPTOMS: $Z=-0.75, P=0.453$; ECG: $Z=-0.98$,
$P=0.327$ ) and the Egger's test (SYMPTOMS: $t=-1.15$, $P=0.301$; ECG: $t=-0.70, P=0.536)$ also found no statistically significant publication bias.

3.8. Adverse Events. Three studies [30, 32, 35] of seven included studies did not report AEs. The AEs among 155 participants in the studies with AE reports were mainly headache and dizziness. A study [29] reported that the AE incidence of 
TABLE 4: Metaregression of basic characteristics of RCTs and ORs of SYMPTOMS and ECG outcomes.

\begin{tabular}{|c|c|c|c|c|c|c|}
\hline Log OR & No. of RCTs & No. of participants & Factor tested & Coefficient & $t$ & $P$ \\
\hline \multirow{7}{*}{ SYMPTOMS } & \multirow{7}{*}{7} & \multirow{7}{*}{550} & Publication year & 0.0200 & 0.43 & 0.685 \\
\hline & & & No. of authors & -0.2106 & -1.63 & 0.165 \\
\hline & & & Trail date & 0.5646 & 1.00 & 0.365 \\
\hline & & & Sample size & 0.0069 & 1.52 & 0.190 \\
\hline & & & Comparable & -0.6369 & -1.38 & 0.227 \\
\hline & & & AEs & -0.4280 & -0.89 & 0.412 \\
\hline & & & M score & -0.4829 & -1.56 & 0.180 \\
\hline \multirow{7}{*}{ ECG } & \multirow{7}{*}{5} & \multirow{7}{*}{370} & Publication year & 0.0097 & 0.26 & 0.809 \\
\hline & & & No. of authors & -0.1796 & -1.46 & 0.239 \\
\hline & & & Trail date & 0.4354 & 0.83 & 0.469 \\
\hline & & & Sample size & 0.0073 & 1.10 & 0.354 \\
\hline & & & Comparable & -0.3195 & -0.64 & 0.565 \\
\hline & & & AEs & -0.0911 & -0.16 & 0.886 \\
\hline & & & M score & -0.4354 & -0.83 & 0.469 \\
\hline
\end{tabular}

Metaregression was conducted by residual (restricted) maximum likelihood (REML) with Knapp-Hartung modification.

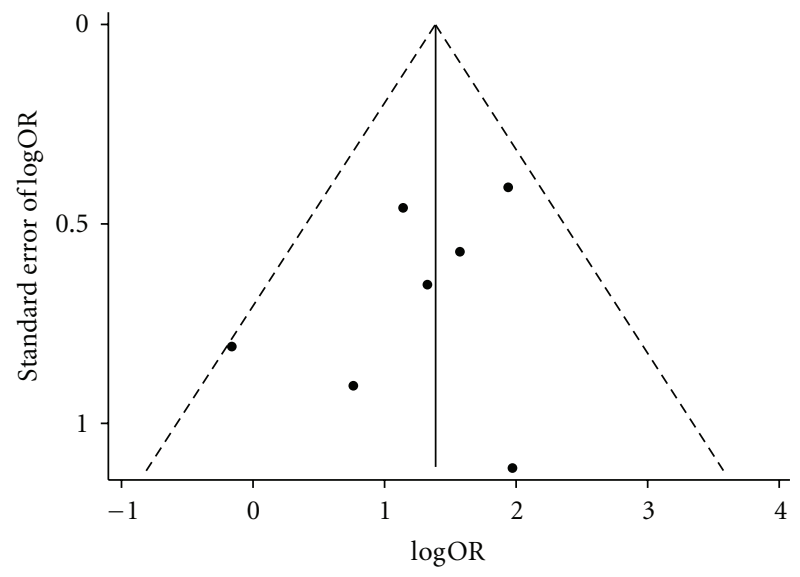

(a)

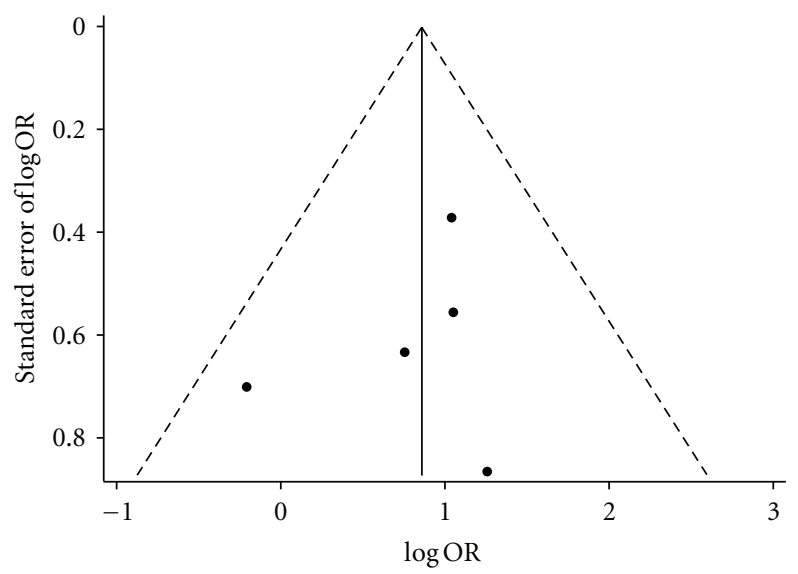

(b)

FIGURE 4: Funnel plots of (a) the included studies with SYMPTOMS data and (b) the included studies with ECG data.

ISDN was $22.22 \%(4 / 18)$ and the AE incidence of XXK was $12.50 \%(2 / 16)$. Another study [34] reported that of the AE incidence of ISDN was $13.33 \%(4 / 30)$ and the AE incidence of XXK was $0 \%(0 / 30)$. The third study [33] reported that the AE incidence of ISDN was 28.79\% (19/66) and the AE incidence of XXK was $0 \%(0 / 66)$. The last study [31] reported no AE for all participants. The overall AE incidence of XXK at $1.30 \%(2 / 155)$ was lower than that of ISDN at $17.42 \%$ (27/155).

\section{Discussion}

This study provides a PRISMA-compliant systematic review and meta-analysis based on the RCTs of XXK and ISDN to evaluate the efficacy in treating angina pectoris. Seven RCTs with 550 participants were found eligible and thus included. Overall ORs of SYMPTOMS and ECG were 4.11 and 2.37, respectively and were statistically significant $(P<0.05)$. Subgroup analysis, sensitivity analysis, and metaregression on various parameters showed this positive evidence to be rozbust. This meta-analysis found that XXK seems to be more effective than ISDN in treating angina pectoris.

This systematic review is comprehensive, reliable, and PRISMA-compliant. In this meta-analysis, (a) a comprehensive search for RCTs published between 1989 and 2011 were performed; (b) only the studies with XXK and ISDN as single-drug interventions were included; (c) subgroup analysis, sensitivity analysis, and metaregression were performed according to the basic characteristics of included studies; (d) publication bias was assessed not only by funnel plots but also by the Begg's test and Egger's test.

This study indicated XXK seems to be more effective than ISDN in treating angina pectoris. The active components of XXK are steroidal saponin glycosides, among which Saponin DP $1-8$ is a major component [37]. XXK were able to 
(a) maintain the activity of $\mathrm{Ca}^{2+}$-ATP enzyme and $\mathrm{Na}^{+}$$\mathrm{K}^{+}$-ATP enzyme through free radicals removal; (b) protect myocardial cells from ischemia and maintain the integrity of myocardial cell membrane; (c) protect myocardial cells through dilated coronary arteries, reduced coronary resistance, increased coronary blood flow, improved blood and oxygen supply to myocardium; (d) improve myocardial ischemia symptoms through reducing myocardial oxygen consumption and enhancing tolerance of myocardium to hypoxia $[38,39]$.

A major limitation of this systematic review, like other systematic reviews on Chinese RCTs [16], is the quality of included studies. Only $6.8 \%$ RCTs on 20 common diseases published from January 1994 to June 2005 are authentic RCTs conducted in China National Knowledge Infrastructure electronic database [40]. Most of the included studies scored only 2 at the Jadad scale, which is a scale ranged between 0 and 5. Neither allocation concealment nor blinding was mentioned in the included studies. Four out of seven included studies scored only 3 at the M scale (ranged between 1 and 7). Although we found no statistically significant difference in the effect sizes between different grades of study quality, further updates of this systematic review should be required to confirm the efficacy of XXK over ISDN.

All studies included in this meta-analysis did not report full outcome measure data. They only reported the results based on the number of participants with different responses to the treatments. Moreover, the follow-up period of all studies was only 28 days; thus, tolerance of ISDN over time would not be estimated. Tolerance of ISDN was not adequately considered in past meta-analyses of (even) western medicine RCTs.

This systematic review included the studies comparing XXK and ISDN and excluded the studies with combined drugs. The dosages of XXK were in accordance with recommended dosages (two pills each time, three times a day) of the manufacturer. Daily dosages of ISDN ranged from $30 \mathrm{mg}$ to $60 \mathrm{mg}$. The guideline of ACC/AHA 2002 suggested the oral dosage range of ISDN to be $5-80 \mathrm{mg}$ each time twice or three times a day. A previous study [41] found that high dosages of ISDN were not better than low dosages of ISDN in treating angina pectoris. The interpretation of the trial results in this systematic review would not be complicated by the combined use of DSP or ISDN with other drugs.

Incidence of AEs due to XXK (1.30\%) was lower than those due to ISDN (17.42\%). All seven included studies did not report AEs in a standard format. Three studies did not report AEs at all. Thus, the safety of XXK could not be thoroughly evaluated although it is well appreciated in the research community that RCTs should report AEs properly [42].

\section{Conclusion}

The meta-analysis of eligible but limited RCTs demonstrates that XXK seems to be more effective than ISDN in treating angina pectoris. Further RCTs of high quality are warranted to be conducted for update of the results of this metaanalysis.

\section{Conflict of Interests}

The authors declare no competing interest.

\section{Authors' Contributions}

Y. Jia and S. Leung designed the study. C. Chen and Y. Jia searched databases. C. Ng and Y. Jia extracted data and assessed the study quality. Y. Jia and C. Ng performed metaanalysis in parallel and independently. C. Chen checked and repeated the data analysis. S. Leung and Y. Jia wrote the paper. All authors read and approved the final manuscript.

\section{Acknowledgment}

The research was supported by a Research Grant from the University of Macau "Open systematic reviewing of clinical trials” (MYRG190 (Y1-L3)-ICMS11-LSW).

\section{References}

[1] E. Rapaport, R. Bernard, and E. Corday, "Nomenclature and criteria for diagnosis of ischemic heart disease. Report of the Joint International Society and Federation of Cardiology/World Health Organization Task Force on standardization of clinical nomenclature," Circulation, vol. 59, no. 3, pp. 607609, 1979.

[2] Scottish Intercollegiate Guidelines Networks, Management of Stable Angina, A national clinical guideline, no. 96, 2007.

[3] R. J. Gibbons, J. Abrams, P. C. Deedwania et al., "ACC/AHA practice guidelines-ACC/AHA 2002 guideline update for the management of patients with chronic stable angina task force on practice guidelines (committee to update the 1999 guidelines)," Circulation, vol. 107, no. 1, pp. 149-158, 2003.

[4] L. Cui and P. A. Zhou, "A new therapy named transmyocardial laser revascularization for treat ischemic heart disease," Chinese Journal for Clinicians, vol. 28, no. 9, pp. 59-60, 2000.

[5] J. Abrams, "Beneficial actions of nitrates in cardiovascular disease," The American Journal of Cardiology, vol. 77, no. 13, pp. 31-37, 1996.

[6] Z. Liu, W. Zhou, R. Wang, and Z. Zhou, "Efficacy evaluation on di'ao xinxuekang last decade," China Journal of Traditional Chinese Medicine and Pharmacy, vol. 19, no. 10, pp. 620-622, 2004.

[7] Chinese Pharmacopoeia Committee, Chinese Pharmacopoeia, 9th edition, 2010.

[8] J. Xu and Y. Wang, "Clinical efficacy evaluation of di' ao capsule treatment for silent myocardial ischemia in patients of cardiac disease," Shenzhen Journal of Integrated Traditional Chinese and Western Medicine, vol. 1, pp. 25-27, 2006.

[9] X. Jin, "Fifty patients with ischemic heart disease treated with di'ao xinxuekang," Medical Information, vol. 24, no. 6, p. 163, 2011.

[10] Z. Huang, "Evaluation the efficacy of di'ao xinxuekang capsule treating ischemic heart disease angina pectoris," Medical Forum, vol. 1, pp. 24-25, 2009.

[11] Chinese Medicine Experts Group of European Federation of Chinese Medicine Societies, "Meta-analysis of di'ao xinxuekang capsule in treating ischemic heart disease," Chinese Community Doctors, vol. 16, p. 15, 2010. 
[12] Q. Zhu and L. Guojuan, "The main points to distinguish true and false di'ao xinxuekang capsule," Chinese Magazine of Clinical Medicinal Professional Research, vol. 7, pp. 995-996, 2008.

[13] J. Meng, "Tasly Pharmaceuticals: the leader of modern traditional Chinese medicine," China Pharmaceutical, vol. 18, no. 3, pp. 239-240, 2007.

[14] X. Deng, "Efficacy evaluation of di'ao xinxuekang and isosorbide dinitrate in treating angina pectoris with different times," Modern Diagnosis \& Treatment, vol. 7, no. 3, pp. 172-173, 1996.

[15] Z. Wang, "Efficacy evaluation of combined traditional Chinese medicine and western medicine in treating 60 participants with angina pectoris," China New Medicine Forum, vol. 9, pp. 18-19, 2007.

[16] Y. Jia, F. Huang, S. Zhang, and S. Leung, "Is danshen (Salvia miltiorrhiza) dripping pill more effective than isosorbide dinitrate in treating angina pectoris? A systematic review of randomized controlled trials," International Journal of Cardiology. In press.

[17] D. Moher, A. Liberati, J. Tetzlaff, and D. G. Altman, "The PRISMA Group. Preferred reporting items for systematic reviews and meta-analyses: the PRISMA Statement," Plos Medicine, vol. 89, no. 9, pp. 873-880, 2009.

[18] A. R. Jadad, R. A. Moore, D. Carroll et al., "Assessing the quality of reports of randomized clinical trials: is blinding necessary?" Controlled Clinical Trials, vol. 17, no. 1, pp. 1-12, 1996.

[19] J. Higgins and S. Green, "Assessing risk of bias in included studies," in Cochrane Handbook for Systematic Reviews of Interventions Version 5.0.1, J. P. T. Higgins and S. Green, Eds., chapter 8, 2008, updated September 2008.

[20] T. Lewis, "PROC LOGISTIC: the logistics behind interpreting categorical variable effects," Statistical Data Analysis, pp. 1-7, 2007.

[21] L. Breierova and M. Choudhari, "An introduction to sensitivity analysis," Massachusetts Institute of Technology, vol. 10, pp. 41-107, 2001.

[22] J. P. T. Higgins and S. G. Thompson, "Quantifying heterogeneity in a meta-analysis," Statistics in Medicine, vol. 21, no. 11, pp. 1539-1558, 2002.

[23] J. Sterne and M. Egger, "Funnel plots for detecting bias in meta-analysis: guidelines on choice of axis," Journal of Clinical Epidemiology, vol. 54, no. 10, pp. 1046-1055, 2001.

[24] C. B. Begg and M. Mazumdar, "Operating characteristics of a rank correlation test for publication bias," Biometrics, vol. 50, no. 4, pp. 1088-1101, 1994.

[25] M. Egger, G. D. Smith, M. Schneider, and C. Minder, "Bias in meta-analysis detected by a simple, graphical test. Increase in studies of publication bias coincided with increasing use of meta-analysis," British Medical Journal, vol. 316, pp. 629-634, 1997.

[26] S. G. Thompson, J. P. T. Higgins, and R. Way, "How should meta-regression analyses be undertaken and interpreted?" Statistics in Medicine, vol. 21, no. 11, pp. 1559-1573, 2002.

[27] Review Manager (RevMan) [Computer program], Version 5.1. Copenhagen, Denmark, The Nordic Cochrane Centre, the Cochrane Collaboration, 2011.

[28] R Development Core Team, "R: a language and environment for statistical computing," reference index version 2.12.0. R Foundation for Statistical Computing, Vienna, Austria, 2005, http://www.r-project.org/.
[29] K. L. Zeng, H. J. Zeng, W. Gong, G. Shi, W. Cheng, and W. Luo, "Isosorbide mononitrate and di'ao xinxuekang in treating angina pectoris," Journals of Naval Medical Colleges, vol. 18, no. 4, pp. 245-246, 1996.

[30] H. P. Dai, "Efficacy evalution of di'ao xinxuekang treating 60 participants with ischemic heart disease," The Medical Journal of Industrial Enterprise, vol. 13, no. 4, p. 19, 2000.

[31] Q. Y. Hu, "Efficacy evalution of di'ao xinxuekang treating 43 participants with ischemic heart disease angina pectoris," Chinese Journal of Primary Medicine and Pharmacy, vol. 16, no. 10, pp. 1882-1883, 2009.

[32] S. H. Qu, "Efficacy evalution of di'ao xinxuekang treating 30 participants with ischemic heart disease," Journal of Changchun University of Traditional Chinese Medicine, vol. 22, no. 2, p. 20, 2006.

[33] D. J. Shen, "Efficacy evalution of di'ao xinxuekang treating participants with ischemic heart disease," Journal of Guilin Medical College, vol. 3, no. 10, pp. 309-310, 1997.

[34] Y. Q. Li and X. Zhang, "Efficacy evalution of di'ao xinxuekang combined with hyperbaric oxygen in treating 30 participants with type II diabetes complicated with ischemic heart disease," Youjiang Medical Journal, vol. 28, no. 2, pp. 81-82, 2000.

[35] G. H. Zhu, Q. Liu, X. Zhang, X. Li, and Y. Wei, "Clinical efficacy evalution of di'ao xinxuekang treating 30 participants with stable angina pectoris," China Modern Doctor, vol. 45, no. 15, pp. 120-121, 2007.

[36] G. Wang, B. Mao, Z. Xiong et al., "The quality of reporting of randomized controlled trials of traditional Chinese medicine: a survey of 13 randomly selected journals from mainland China," Clinical Therapeutics, vol. 29, no. 7, pp. 1456-1467, 2007.

[37] H. Y. Lin, Y. J. Lou, H. G. Lou, and H. H. Wu, "Protective effect of total flavones from Elsholtzia blanda (TFEB) on myocardial ischemia induced by coronary occlusion in canines," Journal of Ethnopharmacology, vol. 94, no. 1, pp. 101-107, 2004.

[38] X. J. Yang, Y. B. Yang, and J. H. Luo, "The pharmacological action and clinical application of di'ao xinxuekang," Pharmaceutical Industry Journal of Shandong, vol. 22, no. 2, pp. 30-32, 2003.

[39] National Research Center of Natural Drug and Engineering Technology, "The pharmacological action and clinical application of the principal components in di'ao xinxuekang," Chinese Community Doctors, vol. 30, p. 16, 2010.

[40] T. Wu, Y. Li, Z. Bian, G. Liu, and D. Moher, "Randomized trials published in some Chinese journals: how many are randomized?" Trials, vol. 10, article 46, 2009.

[41] U. Thadani, H. L. Fung, A. C. Darke, and J. O. Parker, "Oral isosorbide dinitrate in the treatment of angina pectoris. Doseresponse relationship and duration of action during acute therapy," Circulation, vol. 62, no. 3, pp. 491-502, 1980.

[42] X. Wang, W. Da, H. Wang, and J. Xu, "Problems in adverse drug reaction reports and suggestions for improvement," Shanghai Medical \& Pharmaceutical Journal, vol. 27, no. 5, pp. 215-216, 2006. 


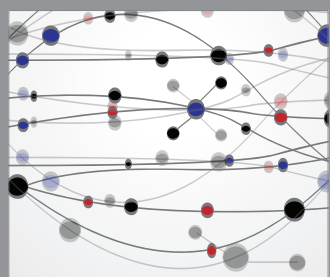

The Scientific World Journal
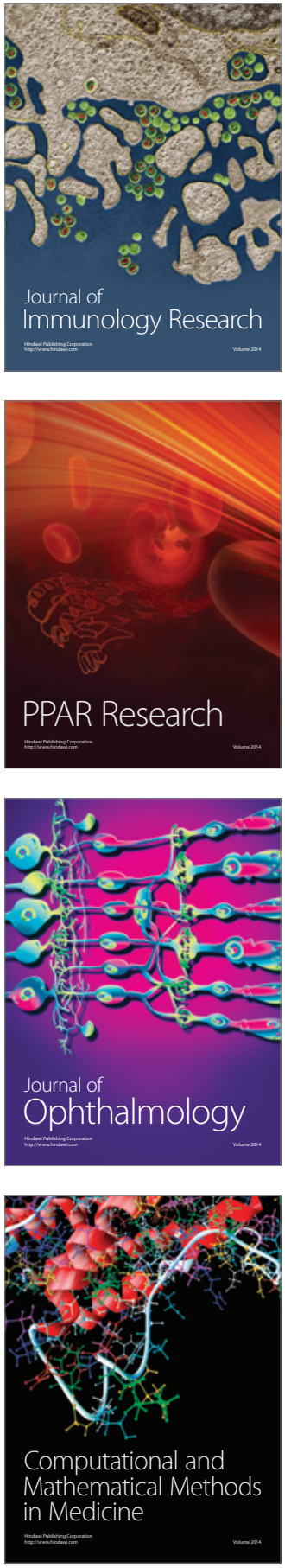

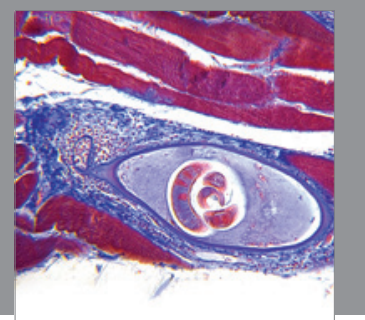

Gastroenterology

Research and Practice
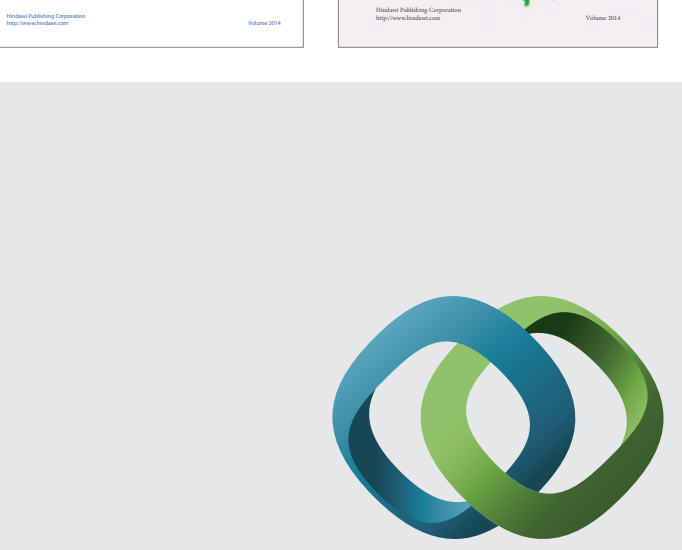

\section{Hindawi}

Submit your manuscripts at

http://www.hindawi.com
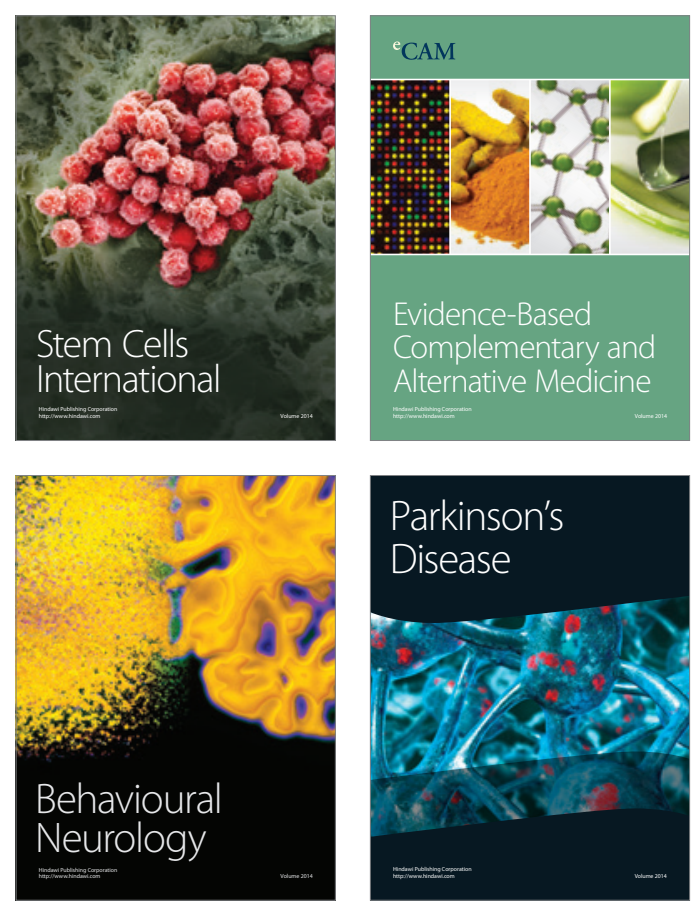

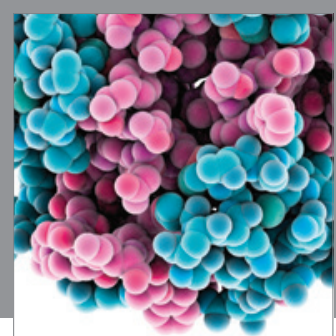

Journal of
Diabetes Research

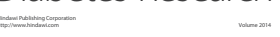

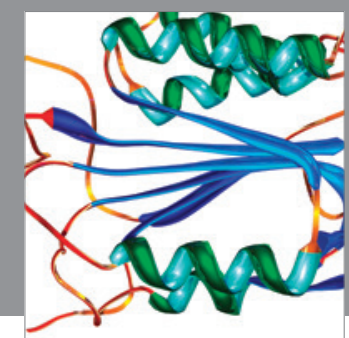

Disease Markers
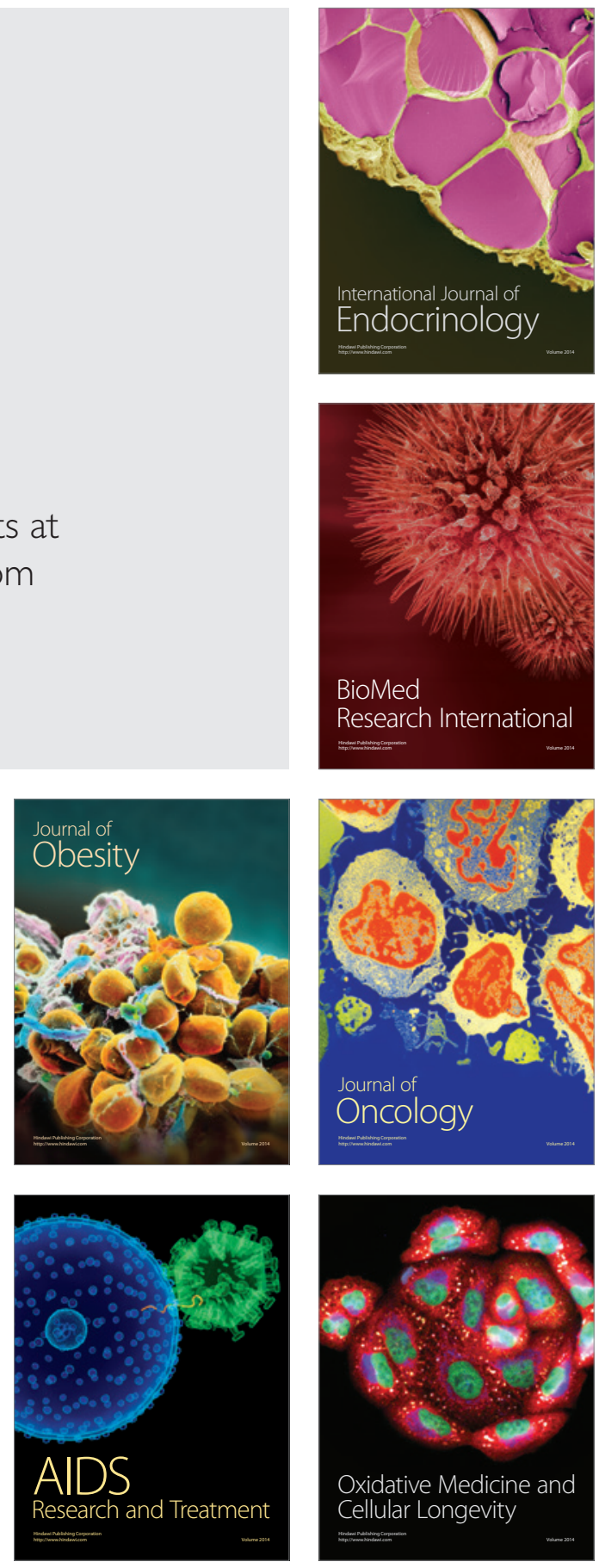